\title{
Familial autosomal recessive rigid spine syndrome with neurogenic facio-scapulo-peroneal muscle atrophy
}

\author{
Laura Palmucci, Tiziana Mongini, Carlo Doriguzzi, Michele Maniscalco, Davide Schiffer
}

\begin{abstract}
Two sisters and a first cousin presented with rigid spine and facio-scapuloperoneal muscle atrophy. The patients belonged to a family with two firstcousin marriages. Electromyography, muscle and nerve biopsy showed neurogenic muscle atrophy without peripheral nerve involvement. Follow up did not show progression of the disease. This is the first observation of an association of neurogenic facio-scapulo-peroneal and rigid spine syndrome. The double first-cousin marriage suggests autosomal recessive inheritance.
\end{abstract}

Muscle involvement with facio-scapuloperoneal distribution may be found in different pathological conditions, including myopathies and neurogenic atrophies. ${ }^{1-5}$ Whereas facio-scapulo-peroneal myopathy is relatively common, especially as a form of the autosomal dominant facio-scapulo-humeral muscular dystrophy (Landouzy-Dejerine type), neurogenic forms are much less frequent. Among them both neuropathies and motor neuron diseases are reported ${ }^{34-14}$ and these are usually classified as scapulo-peroneal syndrome: facial involvement is only occasionally reported. ${ }^{67}$ The so-called "rigid spine syndrome," first described by Dubowitz, ${ }^{15}$ is a rare pathological condition characterised by limitation in flexion of the spine and myopathic changes in skeletal muscle. Most reported cases are sporadic, but autosomal dominant familial cases have also been described associated with scapuloperoneal myopathy and severe cardiac involvement. ${ }^{1617}$ Three familial cases of associated neurogenic facio-scapulo-peroneal and rigid spine syndromes, with autosomal recessive inheritance are described.

Paolo Peirolo Centre for Neuromuscular Diseases, Clinica Neurologica II, Università di Torino, Turin, Italy L Palmucci T Mongini C Doriguzzi M Maniscalco D Schiffer

Correspondence to: Dr Palmucci, Clinica Neurologica II via Cherasco 15 I 10126 Torino, Italy.

Received 16 August 1989 and in revised form 21 February 1990. Accepted 23 May 1990

\section{Case report}

The cases belong to a family with two firstcousin marriages (fig 1).

\section{Case 1 (IV-2)}

This was a female born after a normal pregnancy and delivery. Normal psychomotor development was referred. The patient was unable to touch her toes bending forward. At the age of two years the patient was noted to be walking on tiptoes and she had bilateral tenotomy. She was first referred to us at the age of 12 years.

Clinical examination showed a thin girl with a block of the elbows in semiflexion.
There was hyperlordosis and marked limitation of flexion of the thoracolumbar spine (fig 2a). Weakness of the orbicularis oculi, orbicularis oris and buccinator muscles was evident. She was unable to screw up her eyes or whistle. There was mild hypotrophy of the shoulder girdle with slight proximal weakness and winging of the scapulae. The sternocleidomastoid muscle was not involved. The lower girdle was not affected; there was neither a waddling gait nor Gowers' sign. Atrophy of the legs was present. There was slight weakness of the gastrocnemius and severe impairment of the peroneal and tibialis anterior with stepping gait: dorsiflexion of the feet was impossible. Reflexes were absent in the upper limbs and weak in the lower limbs. All forms of sensation were normal.

Serum creatine kinase (CK) was $290 \mathrm{U} / 1$ (nv up to 170), ECG and echocardiography were normal. Electromyography (EMG) of the deltoid, trapezius, sovraspinatus, infraspinatus, tibialis anterior and peroneal muscles showed a neurogenic pattern with increased amplitude and duration of motor unit potentials and reduced recruitment. No fibrillations were observed. Motor and sensory conduction velocities of the median, ulnar, common peroneal and sural nerves were normal.

The patient was seen again six years later and did not show any progression of the disease.

\section{Case 2 (IV-4)}

This was the male cousin of case 1 who had a healthy sister. He was born after a normal pregnancy and delivery. Normal motor development was referred, but he was not able to touch his toes bending forward. At the age of two years the patient had bilateral tenotomy, because of walking on tiptoes. He was first referred to us at the age of nine years.

I

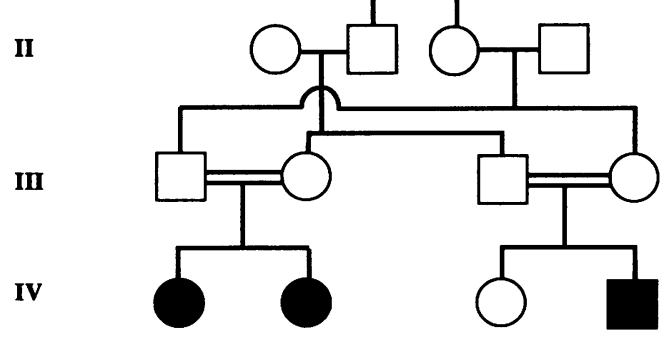

Figure 1 Family tree. 

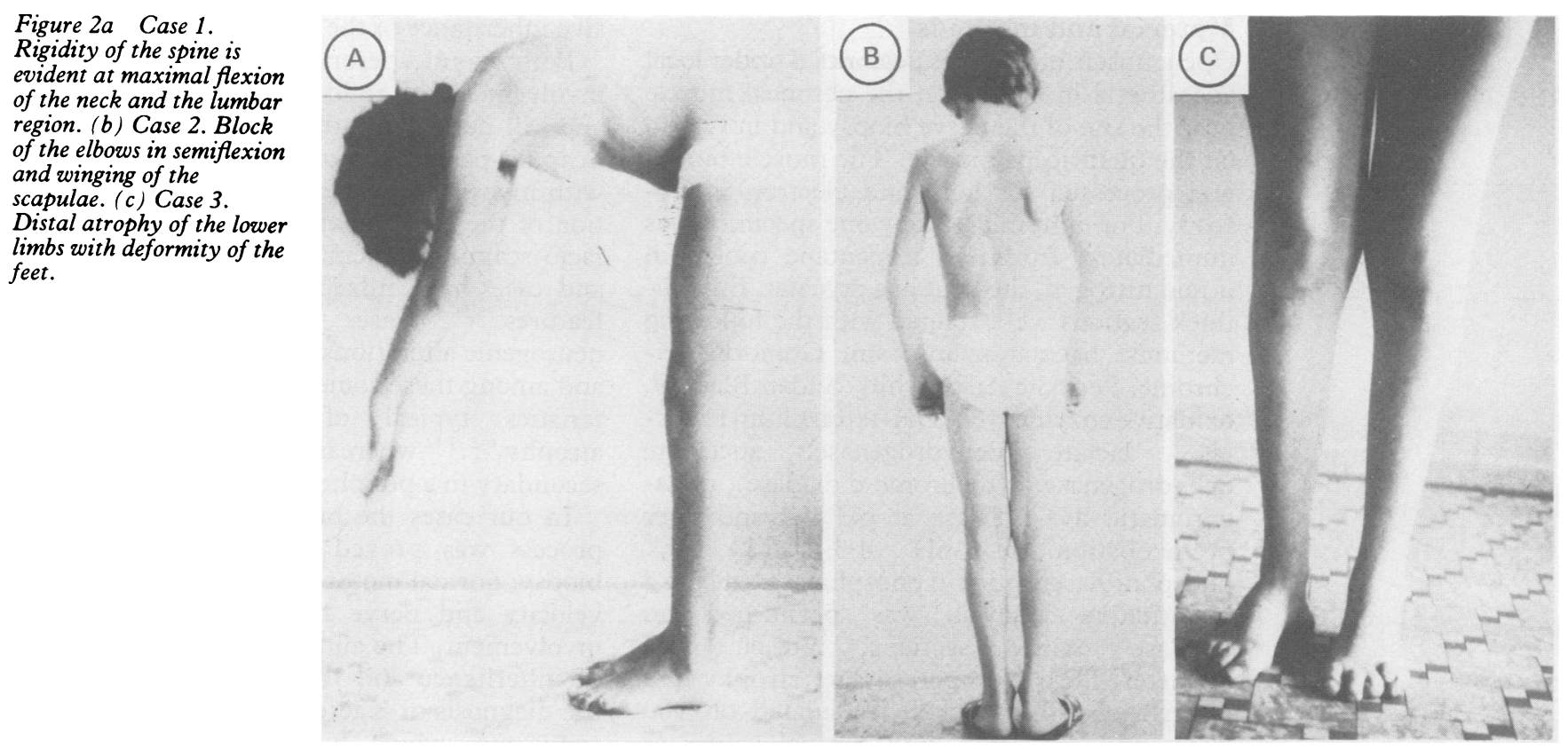

Neurological examination showed rigid spine, limitation of the extension of the elbows with flexion contractures (fig 2b), slight weakness of the orbicularis oculi, orbicularis oris and buccinator muscles; there was slight weakness and wasting of the shoulder girdle with winging of the scapulae. In the lower extremities proximal muscles were spared, whereas distal muscles showed wasting and weakness: dorsiflexion of the feet was impaired. Reflexes were absent in the upper limbs and weak in the lower limbs. Sensation was normal.

Serum CK was 259 U/1, ECG and echocardiography were normal. EMG of the deltoid, trapezius, sovraspinatus, infraspinatus, tibialis anterior and peroneal muscles showed a neurogenic pattern with increased amplitude and duration of motor unit potentials and
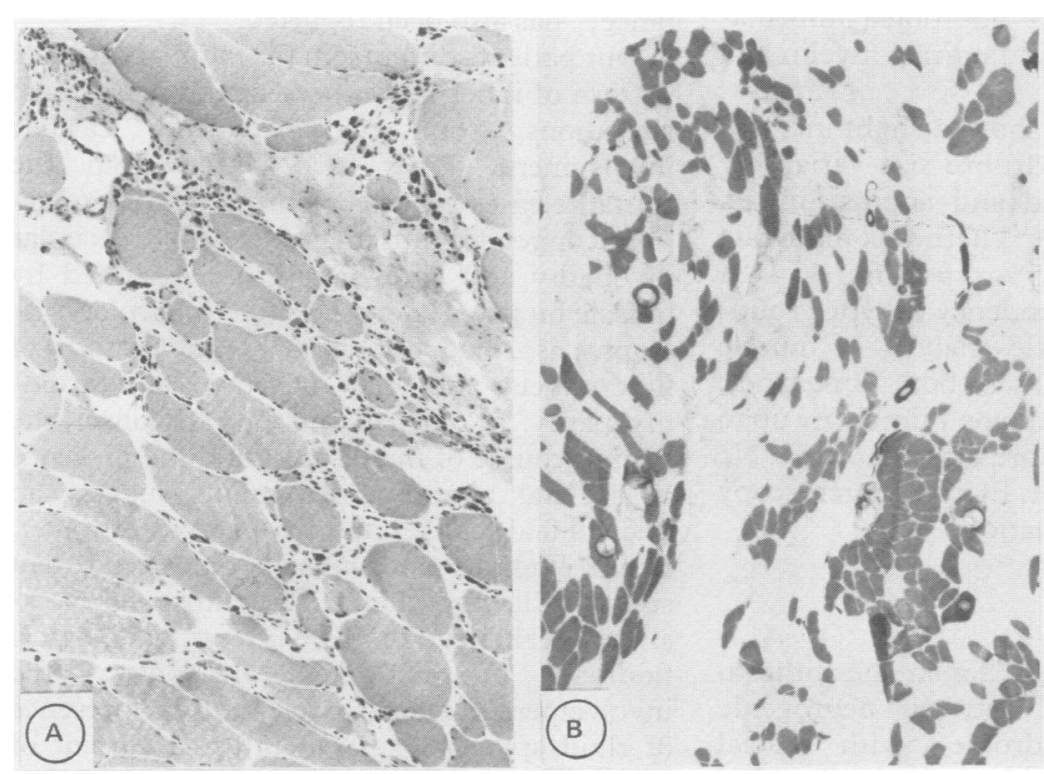

Figure 3 Muscle biopsy of Case 3. (a) Fibre size variability with many small fibres in clusters. $H E 70 \times$. (b) Tendency to type grouping. Metachromatic dye-ATPase $p H$ $4 \cdot 340 \times$. decreased recruitment. Spontaneous activity was absent. Conduction velocities of the median, ulnar, common peroneal and sural nerves were normal.

The patient was re-examined six years later and showed no progression of the disease.

\section{Case 3 (IV-1)}

This was the elder sister of case 1 and the cousin of case 2 and came to our observation only six years after the other two cases, when she was 21 years old. She was born with a bilateral clubfoot. She began to walk at 18 months. She was not able to whistle or screw up her eyes and could not touch her toes bending forward. No progression of the disease was brought to our attention. flexion of the thoracolumbar spine and deformities of the feet with hammer toes. Function of the orbicularis oculi, orbicularis oris and buccinator was impaired. Winging of the scapulae was observed in association with moderate weakness and wasting of the shoulder girdle muscles. In the lower limbs severe wasting and weakness were evident in the legs (fig 2c) and associated with marked reduction of the dorsiflexion of the feet and stepping gait. Moderate involvement of proximal muscles with positive Gowers' sign was also present. Reflexes were weak in the upper limbs and knees and absent at the ankles. All forms of sensation were normal.

Serum CK was $310 \mathrm{U} / 1$. ECG and echocardiography were normal. EMG of the deltoid, trapezius, sovraspinatus, infraspinatus, vastus lateralis and medialis, gastrocnemius, tibialis anterior and peroneal muscles showed high potentials reduced in frequency, decreased recruitment of motor units, especially in the tibialis anterior and peroneal muscles. No spontaneous activity was present. Motor and sensory conduction velocities of median, ulnar, common peroneal and sural nerves were normal.
Examination showed marked limitation of 


\section{Material and methods}

Open muscle biopsy was performed under local anaesthesia in Case 2 on the peroneal muscle near the site of the nerve biopsy and in Case 3 on the quadriceps muscle. The muscle biopsy was processed for light and electron microscopy. For light microscopy one specimen was immediately frozen in isopentane cooled in liquid nitrogen, then cut in a cryostat. Eight $\mu$ thick sections were stained with the following methods: haematoxylin-eosin, Gomori's trichrome, Periodic Acid Schiff, Sudan Black B, oxidative enzymes (NADH-tetrazolium reductase, lactate dehydrogenase, succinate dehydrogenase, cytochrome $\mathrm{c}$ oxidase), metachromatic dye-ATPase at $\mathrm{pH} \mathbf{9 . 4}$ and after preincubation at $\mathrm{pH} \quad 4.3$ and $4.5,{ }^{18}$ phosphorylase, and acid phosphatase. In case 2 quantitative analysis was performed on ATPase stained sections; muscle fibre diameter, fibre type percentage, atrophy and hypertrophy factors were calculated on 300 fibres and compared with normal values as previously reported. ${ }^{19}$ One specimen was fixed in glutaraldehyde for electron microscopy, post-fixed in $\mathrm{OsO} 4$ and embedded in Epon 812. Semithin sections were stained with toluidine blue, thin sections were stained with uranyl acetate and lead citrate.

In case 1 and 2 nerve biopsy was performed under local anaesthesia on the peroneal superficial nerve. The specimen was treated for electron microscopy as above.

\section{Results}

Case 1 Nerve biopsy showed normal distribution of myelin fibres. No myelin loss and no sign of degeneration was present. Axons showed normal contents of organelles.

Case 2 Muscle biopsy showed marked increase of connective tissue with abundant vessels and nerve twigs. Muscle fibres were sparse in the tissue, had round shape and variable size with many small fibres. No type differentiation was present. Electron microsocpy did not give additional information. Nerve biopsy showed normal myelin and axons.

Case 3 Muscle biopsy showed slight increase of connective tissue and fibre size variability with many small round and angular fibres, often in clusters (fig 3a). There was moderate type I predominance (55\% type I fibres, $45 \%$ type II fibres), with a tendency to type grouping (fig 3b). Histometric analysis of muscle biopsy confirmed atrophy of both fibre types: atrophy factor was 694 in type I fibres (nv up to 65 ) and 1400 in type II fibres (nv up to 205). No hypertrophy was evident. Electron microscopy added no further information.

\section{Discussion}

The interest of the cases lies in the hitherto unreported association between neurogenic scapulo-peroneal syndrome with facial involvement and rigid spine. Another peculiarity is the unusual double consanguinous marriage, which supports autosomal reces- sive inheritance of the disease.

Both scapulo-peroneal syndrome with facial involvement and rigid spine syndrome are rare and ill-defined pathological entities. ${ }^{1-5}$ 20-22 Scapulo-peroneal syndrome includes cases with myopathic changes, ${ }^{23}{ }^{24}$ raising the question of the relationship of the condition with facio-scapulo-humeral muscular dystrophy, ${ }^{1}$ and cases with mixed myopathic-neurogenic features..$^{25}$ Cases with unquestionable neurogenic alterations have also been reported and among these some presented with characteristics typical of a spinal muscular atrophy, ${ }^{6-81112}$ whereas others were considered secondary to a peripheral neuropathy. ${ }^{1013}$

In our cases the neurogenic nature of the process was proved by EMG and muscle biopsy; normal motor and sensory conduction velocity and nerve biopsy ruled out nerve involvement. The autosomal recessive pattern of inheritance and the early onset excluded the diagnosis of Kaeser type scapulo-peroneal syndrome, which has autosomal dominant inheritance and late onset. ${ }^{6}$ Only a few cases of neurogenic scapulo-peroneal syndrome with early onset are reported. ${ }^{347-911} 12$ They are more often sporadic and only one of them ${ }^{7}$ showed facial involvement; the rigid spine syndrome was not mentioned in these cases.

Rigid spine syndrome $\mathrm{e}^{15}$ is an unusual condition whose nosology is still uncertain. ${ }^{2021}$ Most reported cases are sporadic and affecting males. Familial cases have been reported with autosomal dominant inheritance, in association with myopathic scapulo-peroneal syndrome and cardiomyopathy. ${ }^{16} 17$ Sporadic cases are associated with myopathic muscle changes and are variously classified. In some cases congenital myopathy has been suggested, in particular fibre type disproportion, ${ }^{21} 27$ in others congenital muscular dystrophy 222728 or EmeryDreifuss muscular dystrophy have been suggested. ${ }^{29}$ Histological features of an unclassified vacuolar myopathy are occasionally observed..$^{201130}$ Multicore myopathy, both with autosomal dominant ${ }^{31}$ and recessive inheritance, ${ }^{32}$ has also been reported.

Our patients differ from the familial cases for pattern of inheritance, presence of neurogenic alterations, and absence of cardiac involvement. They also differ from the sporadic cases reported: fibre type disproportion, congenital muscular dystrophy, vacuolar myopathy and core disease are excluded by muscle biopsy; Emery-Dreifuss muscular dystrophy is ruled out also by the pattern of inheritance and the absence of cardiomyopathy. In our patients the early onset, the benign course of the disease, and the presence of skeletal abnormalities might suggest an unclassified congenital myopathy; however, EMG findings and muscle biopsy did not support this hypothesis. The autosomal recessive inheritance, the EMG and histological findings of neurogenic atrophy, the involvement of facial muscles and the presence of rigid spine suggest an unusual variant of both scapulo-peroneal and rigid spine syndrome, confirming the broad spectrum of these clinical entities. 
1 Ricker K, Mertens H-G. The neurogenic scapulo-peroneal syndrome. Europ Neurol 1968;1:257-74.

2 Ricker K, Mertens H-G. The differential diagnosis of the myogenic (facio)-scapulo-peroneal syndrome. Europ myogenic (facio)-scap

3 André JM, André MN, Floquet J, Tridon P, Arnould G. Les amyotrophies scapulo-péronéales (à propos de deux cas de syndrome de Stark-Kaeser). Ann Méd Nancy 1972; 11:925-38.

4 Mercelis R, Demeester J, Martin J-J. Neurogenic scapuloperoneal syndrome in childhood. $J$ Neurol Neurosurg Psychiatry 1980;43:888-96.

5 Munsat TL. Facioscapulohumeral dystrophy and the scapuloperoneal syndrome. In: Engel AG, Banker BO eds. Myology, basic and clinical. New York: McGraw-Hill 1986:1251-66.

6 Kaeser HE. Scapuloperoneal muscular atrophy. Brain 1965; 88:407-18.

7 Emery ES, Fenichel GM, Eng G. A spinal muscular atrophy with scapuloperoneal distribution. Arch Neurol 1968; 18:129-33.

8 Munsat TL. Infantile scapuloperoneal muscular atrophy. Neurology 1968;18:285.

9 Zellweger H, McCormick WF. Scapuloperoneal dystrophy and scapuloperoneal atrophy. Helv Pediat Acta 1968; 23:643-9.

10 Meadows JC, Marsden CD. Scapuloperoneal amyotrophy. Arch Neurol 1969;20:9-12.

11 Schuchmann L. Spinal muscular atrophy of the scapuloperoneal-type. $Z$ Kinderheilk 1970;109:118-23.

12 Negri S, Caraceni T, Cornelio F. A case of scapulo-tibioperoneal syndrome. Electromyographic and histoenzymologic considerations. Europ Neurol 1973;10:31-40.

13 Schwartz MS, Swash M. Scapuloperoneal atrophy with sensory involvement: Davidenkow's syndrome. J Neurol sensory involvement: Davidenkow's sy

14 Hyser CL, Kissel JT, Warmolts JR, Mendell JR. Scapuloperoneal neuropathy: a distinct clinicopathologic entity. J Neurol Sci 1988;87:91-102.

15 Dubowitz V. Pseudo-muscular dystrophy. In: Research in Muscular Dystrophy. Proc Third Symposium. Research Committee of the Muscular Dystrophy Group of Great Britain. London: Pitman Medical, 1965:57-73.

16 Chakrabarti A, Pearce JMS. Scapuloperoneal syndrome with cardiomyopathy: report of a family with autosomal dominant inheritance and unusual features. $J$ Neurol Neurosurg Psychiatry 1981;44:1146-52.

17 Tanaka K, Yoshimura T, Muratani H, Kira J, Itoyama Y, Goto I. Familial myopathy with scapulohumeral distribution, rigid spine, cardiopathy and mitochondrial abnormality. J Neurol 1989;236:52-4.
18 Doriguzzi C, Mongini T, Palmucci L, Schiffer D. A new method for myofibrillar $\mathrm{Ca}++$ ATPase reaction based on the use of metachromatic dyes: its advantages in muscle fibre typing. Histochem 1983;79:289-94.

19 Doriguzzi C, Mongini T, Palmucci L, Gagnor E, Schiffer D. Quantitative analysis of quadriceps muscle biopsy. Results in 30 healthy females. J Neurol Sci 1984;66: 319-26.

20 Van Munster ETL, Joosten EMG, van Munster-Uijtdehaage MAM, Kruls HJA, ter Laak $\mathrm{HJ}$. The rigid spine syndrome. J Neurol Neurosurg Psychiatry 1986;49. 1292-7.

21 Bertini E, Marini R, Sabetta G, et al. The spectrum of the socalled rigid spine syndrome: nosological considerations and report of three female cases. J Neurol 1986;233: 248-53.

22 Banker BQ. Congenital muscular dystrophy. In: Engel AG Banker BQ, eds. Myology. Basic and clinical. New York: McGraw-Hill, 1986:1367-82.

23 Thomas PK, Schott GD, Morgan-Hughes JA. Adult onset scapuloperoneal myopathy. J Neurol Neurosurg Psychiatry 1975:38:1008-15.

24 Yee WC, Hahn AF, Gilbert JJ. Adult onset scapuloperoneal myopathy: diagnostic value of nerve morphometry and multiple muscle biopsies. J Neurol Neurosurg Psychiatry 1988;51:808-13.

25 Takahashi K, Nakamura H, Nakashima R. Scapuloperoneal dystrophy associated with neurogenic changes. J Neurol Sci 1974; 23:575-83.

26 Spalke $G$, Hökendorf $H$, von Roques P. Zur Differentialdiagnose der scapulo-peronealen Amyotrophie. J Neurol 1976;212:253-69.

27 Echenne B, Astruc J, Brunel D, Pages M, Baldet P Martinazzo G. Congenital muscular dystrophy and rigid spine syndrome. Neuropediatrics 1983;14:97-101.

28 Serratrice G, Cros D, Pellissier JF, Gastaut JL, Pouget J. Dystrophie musculaire congénitale. Rev Neurol 1980; Dystrophie

29 Rowland LP, Fetell M, Olarte M, Hays A, Singh N, Wanat FE. Emery-Dreifuss muscular dystrophy. Ann Neurol 1979;5:111-17.

30 Goto I, Muraoka S, Fujiu N, Ohta M, Kuroiwa Y. Rigid spine syndrome: clinical and histological problems. Neurol 1981;236:143-8.

31 Vanneste JAL, Augustijn PB, Stam FC. The rigid spine syndrome in two sisters. J Neurol Neurosurg Psychiatry 1988;51:131-5.

32 Ben Hamida $M$, Hentati $F$, Ben Hamida C Maladie multiminicores au cours d'un syndrome de la colonne raide. Rev Neurol 1987;143:284-9. 